Research Article

\title{
Multislope PI Modeling and Feedforward Compensation for Piezoelectric Beam
}

\author{
Ming Xu (D), Jia-qi Zhang, Cheng Rong, and Jing Ni \\ School of Mechanical Engineering, Hangzhou Dianzi University, Hangzhou, China \\ Correspondence should be addressed to Ming Xu; xumzju@163.com
}

Received 6 March 2020; Accepted 21 April 2020; Published 11 May 2020

Academic Editor: Sylwester Samborski

Copyright (C) 2020 Ming Xu et al. This is an open access article distributed under the Creative Commons Attribution License, which permits unrestricted use, distribution, and reproduction in any medium, provided the original work is properly cited.

\begin{abstract}
The hysteresis nonlinearity greatly reduces the tracking precision of piezoceramic actuators for expected displacement in a highaccuracy positioning system. In order to effectively compensate the hysteresis for piezoelectric ceramics, a novel modeling method, namely, multislope PI (Prandtl-Ishlinskii) was proposed. In view of the minimum mean square error (MSE) criterion, the weights of an improved PI model were identified by the quadratic programming optimization algorithm. For verifying the accuracy of the proposed multislope PI hysteresis model, a feedforward compensation control for piezoceramic beam was achieved. The corresponding experimental system was established, and the displacement tracking experiments were carried out. The results indicated that the mean tracking error was $0.2828 \mu \mathrm{m}$ and within $1 \%$ of full scale, as well as the MSE was $0.3100 \mu \mathrm{m}$. Compared with the conventional PI model, the proposed multislope PI model demonstrated a significant improvement in positioning performance for the piezoceramic beam.
\end{abstract}

\section{Introduction}

The piezoceramics are ideal materials for achieving micronlevel positioning accuracy in high-precision electromechanical drive systems. However, due to the inherent nonlinear characteristics, the tracking accuracy of piezoelectric ceramic actuators for the desired displacement was greatly reduced $[1,2]$. The nonlinearity of piezoelectric ceramics mainly manifests as hysteresis and creep [3], the most important of which is hysteresis nonlinearity [4]. In order to reduce the shortcomings of hysteresis nonlinearity and improve the control accuracy, the feedforward compensation control scheme was generally chosen. This solution required accurate nonlinear hysteresis modeling of piezoelectric ceramics.

There were two main modeling methods for piezoceramic hysteresis, constitutive method and phenomenological method. For the constitutive model method, it was difficult to obtain an accurate model because of its complicated electromechanical coupling relationship $[5,6]$. In contrast, the phenomenological methods built mathematical models by measuring hysteresis nonlinearity and directly performing model fitting. The commonly used phenomenological models were the Bouc-Wen model [7, 8], Preisach model [9], PI model [10], and polynomial model [11]. The PI model has received widespread attention for its theoretical reversibility and independence of rate [12]. The operator in the PI model, such as a stop operator, was usually used to build a mathematical model of a mass-spring-damper system, from which inverse model was easy to obtain [13]. However, due to the inherent characteristics of the PI model, the fitting error cannot be eliminated [10]. Therefore, some studies have tried to improve the PI modeling accuracy and reduce the fitting error. Gu et al. [14] proposed an improved PI model, in which the generalized input function was used to replace the linear input function, which simplified the formula for describing the asymmetric hysteresis characteristics of piezoelectric ceramics. Wei et al. [15] discussed an improved rate-dependent PI operator and established a dynamic model with obvious compensation performance under variable frequency voltage. However, due to the redundancy of operators, the practical application of this method was limited. Different from the abovementioned methods using dynamic weights and thresholds, a dynamic 
envelope function was introduced into the play operator [16]. The hysteresis nonlinearity was described by a set of hysteresis operators, including gain coefficient and inputdependent hysteresis as well as parameter scheduling methods. Ko et al. [17] proposed a direct construction method based on the identification of the inverse generalized PI model, which was not restricted by the constraints of threshold and density function. Because the PI model is simple and does not require inversion model calculation, $\mathrm{Li}$ et al. [18] used a direct inverse hysteresis compensation method based on the improved PI (MPI) model. Dong et al [19] proposed a three-stage PI model. The abovementioned phenomenological modeling methods started from the characteristics of piezoelectric hysteresis curves, established corresponding hysteresis models to fit the hysteresis curves, and reduced the drawbacks of hysteresis nonlinearity on piezoelectric positioning systems. However, the existing methods were often very complicated, as well as involving numerous parameters to be identified, thereby reducing the feasibility of practical applications.

In this paper, a multislope PI modeling method is proposed and experimentally validated. Section 2 describes the experimental system. In Section 3, the differences between the proposed PI and conventional PI models are discussed, and how the improved PI model overcomes the shortcomings of the conventional PI model are given. In Section 4, further comparisons and discussions are implemented.

\section{Experimental System}

The experimental system is shown in Figure 1, which consisted of four parts, including a piezoceramic beam, a high-voltage power supply, a displacement sensor, and a controller. The PZT-5H piezoceramic beam with a length of $80 \mathrm{~mm}$, a width of $15 \mathrm{~mm}$ and a thickness of $0.75 \mathrm{~mm}$, produced by the Shanghai Institute of Ceramics of the Chinese Academy of Sciences, was used to test the hysteresis curve and implemented to feedforward compensation control in this work. The maximum displacement of this PZT-5H beam was $45 \mu \mathrm{m}$. The programmable DC regulated power supply HSPY-1000 with the power of $100 \mathrm{~W}$ was produced by Beijing HSPY Technology Co. Ltd. Its voltage regulation range is $0 \sim 1000 \mathrm{~V}$ and the current regulation range is $0 \sim 0.1 \mathrm{~A}$, with resolutions $10 \mathrm{mV}$ and $1 \mathrm{~mA}$. The model of displacement sensor is KEYENCE LK-H020, with a repeatability accuracy $0.02 \mu \mathrm{m}$ and linearity $\pm 0.02 \%$ F.S. The controller included three parts, a computer, displacement data acquisition, and feedforward compensation algorithm coded by LabVIEW. The displacement data of the piezoceramic beam was acquired by the LK-Navigator software for the KEYENCE laser sensor, and the feedforward compensation method was implemented in LabVIEW. The communication interface between LabVIEW and highvoltage power supply is RS485, with a baud rate of $19200 \mathrm{bps}$.

Figure 2 shows the control scheme of this work. If not otherwise specified, all driving voltage were peak values in this paper. Primarily, it was necessary to perform driving experiments with voltage $0 \mathrm{~V}-150 \mathrm{~V}$ on the PZT-5H

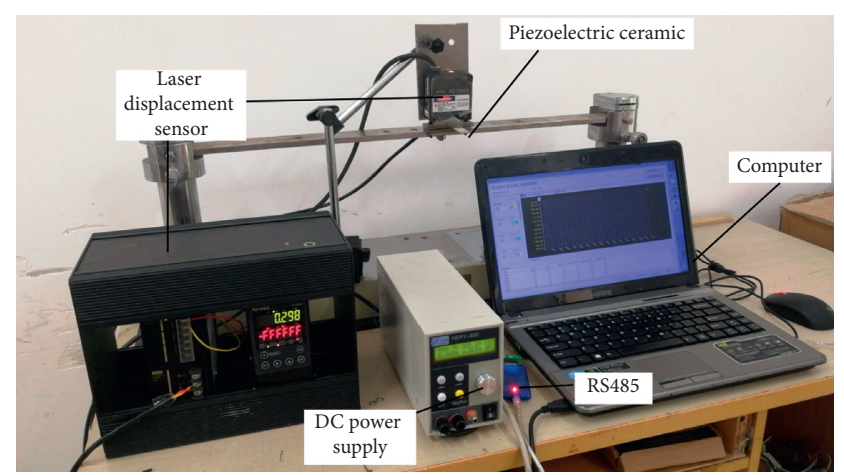

FIgURE 1: Experimental system.

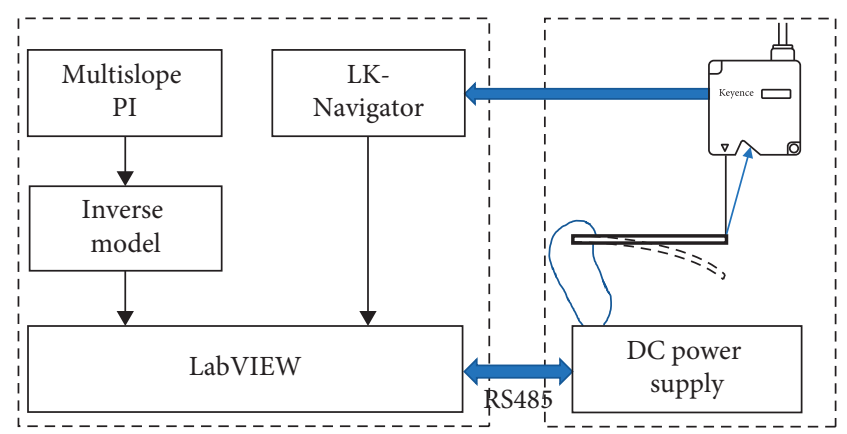

Figure 2: Control scheme of this work.

piezoelectric ceramic beam to obtain and draw the hysteresis curve. Then, a multislope PI mathematical model was designed to fit the hysteresis curve. In order to verify the accuracy of the multislope PI model proposed in the paper, it was also essential to invert the multislope PI model and perform feedforward compensation control on the piezoelectric ceramic beam.

\section{Multislope PI Method}

The novel multislope PI method was actually an improved PI model. Before specifying the proposed multislope PI, the conventional PI model was briefly described.

3.1. Conventional PI Model. The PI model was implemented by multiple play operators superimposing corresponding thresholds, as shown in the following equation:

$$
\left\{\begin{array}{l}
f_{k}(0)=\max \left\{u(0)-r_{k}, \min \left[u(0)+r_{k}, 0\right]\right\}, \\
f_{k}(t)=\max \left\{u(t)-r_{k}, \min \left[u(t)+r_{k}, y(t-T)\right]\right\}, t>0,
\end{array}\right.
$$

where $f_{k}(t)$ is the play operator with threshold $r_{k}, u(t)$ is the driving voltage applied to the piezoelectric ceramics, and $T$ is the sampling period.

As shown in Figure 3, the play operator used for piezoceramic modeling was composed of two stages, the stepup stage and step-down stage, respectively. It was obvious that the single play operator was centrosymmetric, in which 


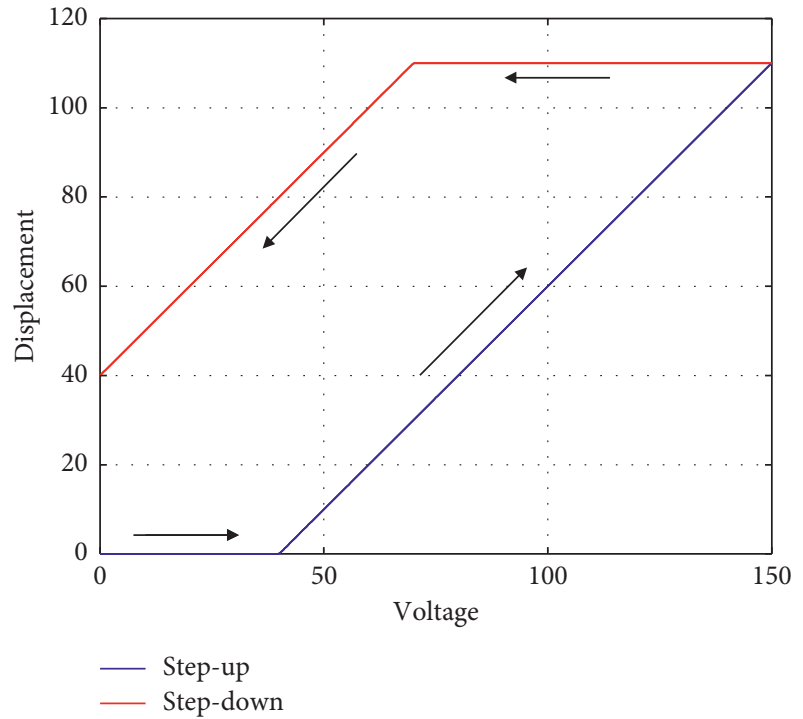

FIgURe 3: An example of the play operator in the PI model.

the starting point of the step-up stage was the coordinate origin, but the finishing point was not the coordinate origin.

The weighted play operator was defined as

$$
\left\{\begin{array}{l}
H_{k}(0)=\omega_{0} \max \left\{u(0)-r_{k}, \min \left[u(0)+r_{k}, 0\right]\right\}, \\
H_{k}(t)=\omega_{k} \max \left\{u(t)-r_{k}, \min \left[u(t)+r_{k}, y(t-T)\right]\right\}, t>0,
\end{array}\right.
$$

where $H_{k}(t)$ is the weighted play operator and $\omega_{k}$ is the value of weight.

The mathematical expression of the PI model was given as follows:

$$
y(t)=\sum_{k=0}^{n} H_{k}(t),=\sum_{k=0}^{n} \omega_{k} \max \left\{u(t)-r_{k}, \min \left[u(t)+r_{k}, y(t-T)\right]\right\},
$$

where $k$ is the number of play operators and $y(t)$ is the displacement output of piezoelectric ceramic.

Equation (3) can also be rewritten as follows:

$$
y(t)=\omega \bullet f(\mathbf{t})^{T},
$$

where the weight matrix $\omega=\left(\omega_{0}, \omega_{1}, \cdots, \omega_{k-1}\right)$ and $f(\mathbf{t})$ is the operator matrix, $f(t)=\left(f_{0}(t), f_{1}(t), \cdots, f_{k-1}(t)\right)$.

3.2. Improved Multislope PI Method. In the PI model, the operators were center-symmetric, as shown in Figure 4(a). However, the hysteresis curve of piezoelectric ceramics was generally not center symmetric. This was the main reason why the PI model was used to describe the hysteresis phenomenon with low accuracy. Besides, when the threshold $r_{k}$ was greater than a certain value $\left(r_{k}>150\right.$ in this work, Figure 4(a)), the PI operator lost the stepdown process, which seriously affected the fitting precision of the PI model to the hysteresis curve. Aiming at the abovementioned problems existing in the conventional PI method, an improved PI model was proposed, in which the multiple slopes were used to replace the

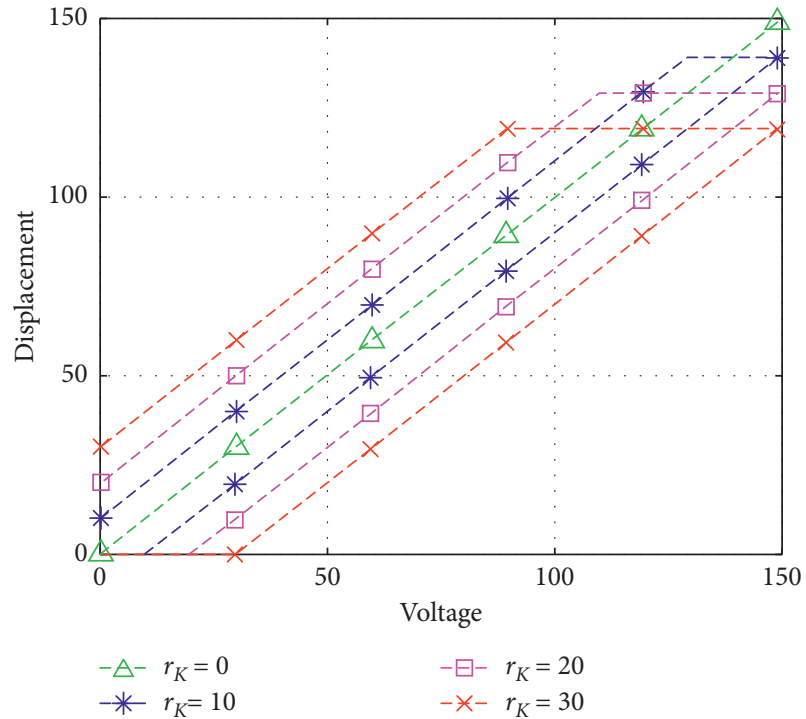

(a)

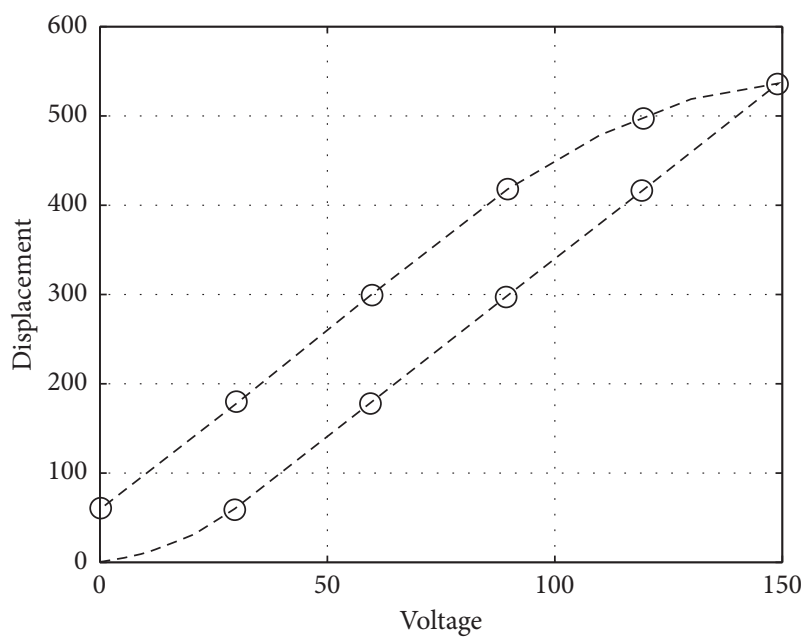

(b)

FIgURE 4: PI model fitting with four operators. (a) Four operators in the PI model. (b) Fitting curve using four operators.

single slope of all operators in the conventional PI model, as well as to reduce the number of operators and model errors.

Figure 4(a) shows four operators $\left(r_{k}=0,10,20,30\right)$, and Figure 4(b) shows the fitting curve using the four operators without considering the weights. As shown in Figure 4(b), since the slopes of all operators were the same, the fitted curve was approximately straight, especially in the middle stages of the step-up and step-down parts, which was quite different from the actual hysteresis curve of the piezoceramic beam. Besides, the return curve did not return to the origin, causing significant model errors.

In order to better explain the improved PI model, the conventional PI model and the improved PI were compared. The driving voltage of the PZT-5H piezoceramic beam during the test was 0 to $150 \mathrm{~V}$. follows:

The play operator in the PI model was described as 


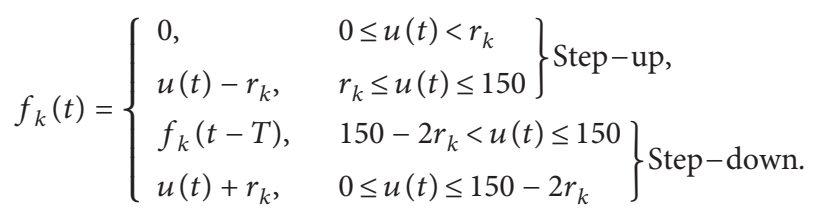

The proposed improved PI model was described by equation (6), where the thresholds were the same as in the conventional PI model.

$$
\left\{\begin{array}{l}
f_{k}(0)=\max \left\{\max \left\{0.8\left[u(0)-r_{k}\right], 1.2 u(0)-r_{k}-30\right\},\right. \\
\left.\min \left\{\min \left[1.2 u(0), 0.8 u(0)+30-0.6 r_{k}\right], y(0)\right\}\right\}, \\
f_{k}(t)=\max \left\{\max \left\{0.8\left[u(t)-r_{k}\right], 1.2 u(t)-r_{k}-30\right\},\right. \\
\left.\min \left\{\min \left[1.2 u(t), 0.8 u(t)+30-0.6 r_{k}\right], y(t-T)\right\}\right\}, t>0 .
\end{array}\right.
$$

The operator comparisons without considering weights between the two PI models are shown in Figure 5, in which the threshold $r_{k}$ was 40 .

For the increase or decrease of the driving voltage, the operator difference between the two PI models was described as follows:

(1) As driving voltage located between $0 \mathrm{~V}$ and $40 \mathrm{~V}$, the two curves represented as the two PI operators coincided with each other

(2) As driving voltage located in increasing stage $(40 \mathrm{~V} \sim 150 \mathrm{~V})$, the concave feature of the operator was needed for decreasing model error, and the broken line represented as the PI operator was replaced by a polyline composed of two straight lines, as shown in Figure 5

(3) As driving voltage located in decreasing stage $(150 \mathrm{~V} \sim 0 \mathrm{~V})$, the horizontal line with a length of 0.5 $r_{k}$ was used as an improved PI operator instead of a

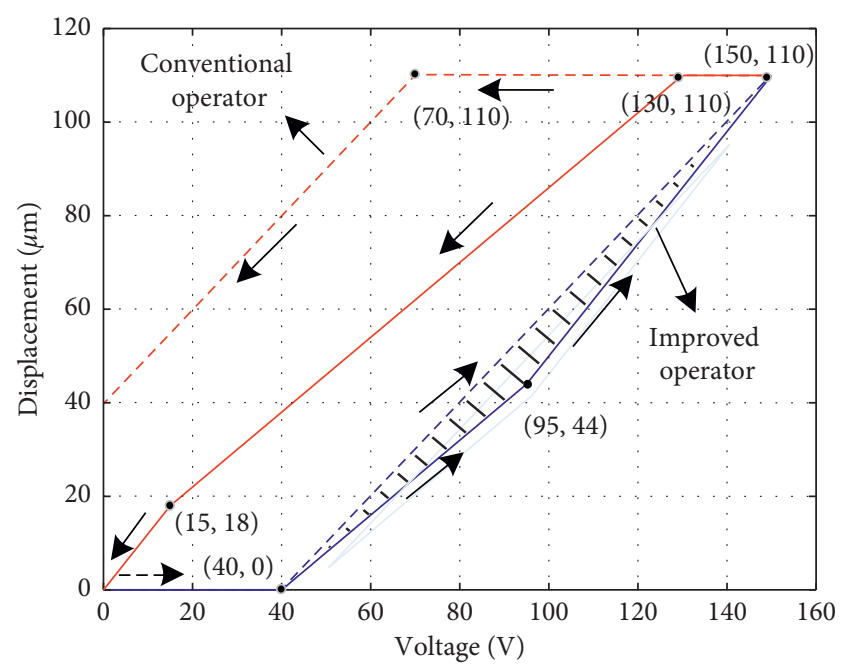

FIgURe 5: Operator comparisons between the two PI models.

horizontal line with a length of $2 r_{k}$, and the returning broken line was also replaced by another polyline consisting of two straight lines, wherein the polyline passed the origin point $(0,0)$.

As shown in Figure 6, the comparisons between the conventional PI model and the improved PI model with the same number of operators and thresholds were illustrated. Each model has four operators and four thresholds, 0, 10, 20, and 30 , respectively. Obviously, the hysteresis curve fitted by the conventional PI method has a great error compared with the actual hysteresis, and the fitted hysteresis curve did not even return to the origin point. Compared with the conventional PI method, the fitting accuracy of the multislope PI model was greatly improved.

The mathematical expressions of improved operators with multiple slopes can also be described as

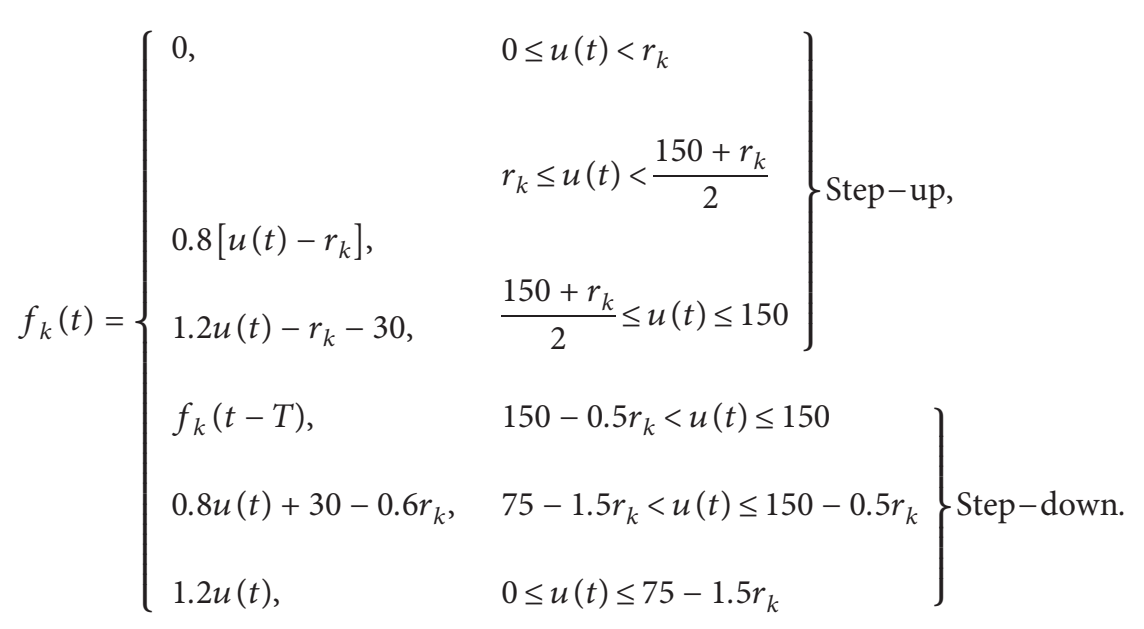

The explanations of equation (7) were given as follows:

(1) As driving voltage located in the increasing stage, there were three straight lines in this stage, Line $a$ with slope 0 , Line $b$ with slope 0.8 , and Line $c$ with slope 1.2. Line $b$ and Line $c$ intersected at the point of $0.5 r_{k}+75$ on the abscissa axis. For the driving voltage 

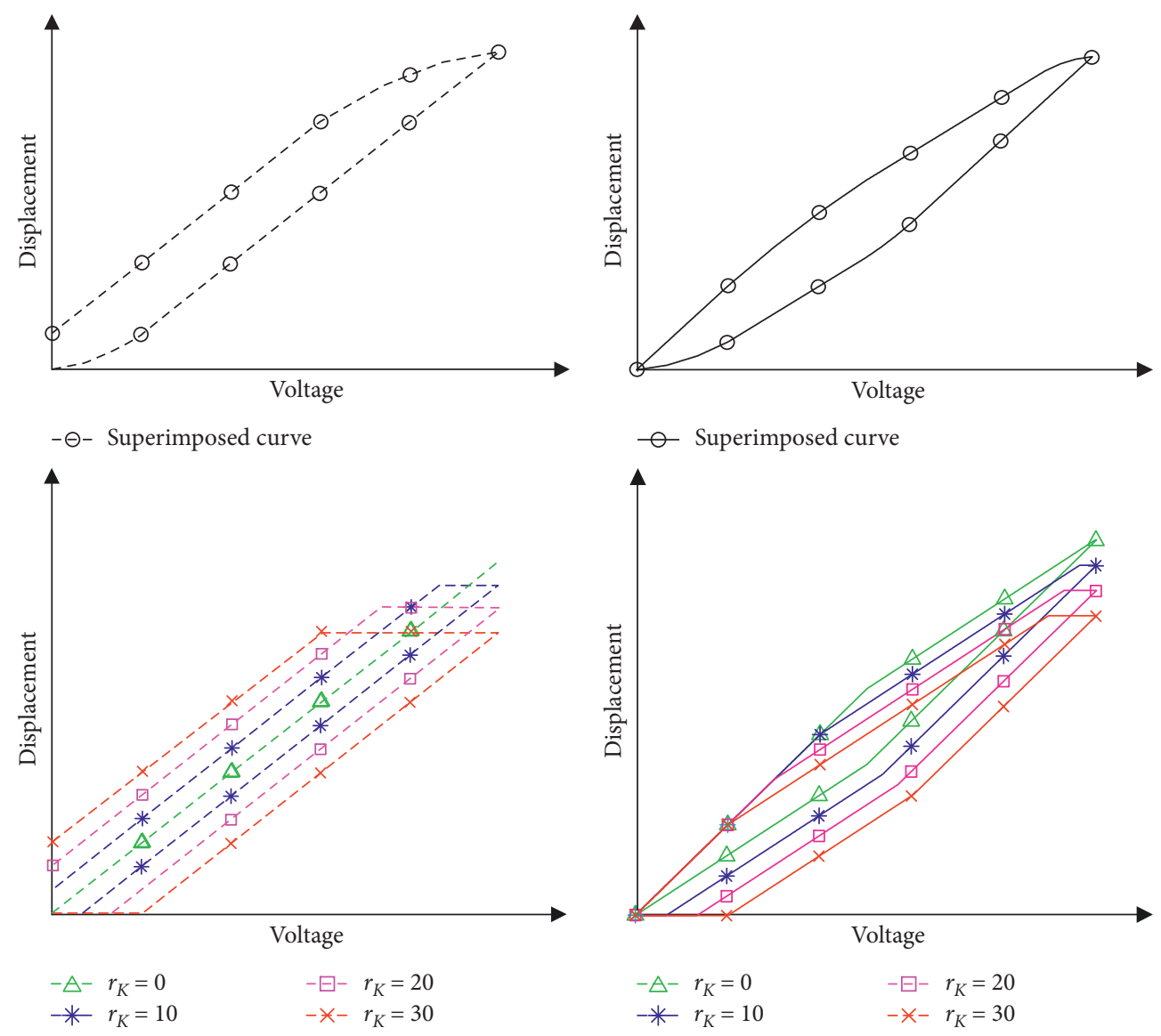

(a)

(b)

FIgURE 6: Comparisons of fitting performance. (a) Conventional PI model. (b) Improved PI model.

$0 \leq u(t) \leq 150$, the threshold value $r_{k} \leq \leq 0.5 r_{k}+75$ $\leq 150$, which implied that the intersection point would always exist with the interval of $\left(r_{k}, 150\right)$, and the operator shape can always show a concave characteristic during the driving voltage increasing phase.

(2) As driving voltage located in the reduction stage, there were also three straight lines in this stage, Line $d$ with slope 0 , Line $e$ with slope 0.8 , and Line $f$ with slope 1.2 , respectively.

In order to ensure the multilateral structure of the operator curve, the inflection point of polyline was designed. In the conventional PI model, considering the influence of the position of inflection point, the slopes of 0.8 and 1.2 were determined by repeated experiments, respectively. If $r_{\mathrm{k}} \leq 50$, then $75-1.5 r_{\mathrm{k}} \geq 0$; that is, the intersection of Line $e$ and Line $f$ was located in the first quadrant. Otherwise, concave-convex features of the fitted curve would not be displayed. Based on the nonredundant principle, four thresholds were determined experimentally in this paper. The four operator thresholds are $0,20,30$, and 40 , which are less than 50 .

3.3. Parameters Identifications. The identification of weight parameters used a sequential quadratic programming algorithm, which was based on the least mean square (LMS) criterion. The optimal solution was obtained by transforming the nonlinear programming into quadratic programming and approximating the optimal value using an iterative method.

The error function was defined as

$$
e(t)=d(t)-y(t)
$$

where $d(t)$ is the expected displacement of the piezoceramic beam.

The mean square error (MSE) was

$$
\varepsilon=\sum_{t=1}^{i} e^{2}(t)=\sum_{t=1}^{i}\left[d^{2}(t)-2 d(t) \cdot y(t)+y^{2}(t)\right],
$$

where $i$ is the number of sampling points.

Assuming the target function is $\delta=1 / 2 \sum_{t=1}^{i} y^{2}(t)-\sum_{t=1}^{i} d(t) \cdot y(t)$, then the vector form can be written as $\min \left\{1 / 2 \omega^{\mathrm{T}} \mathrm{H} \omega+\Phi^{\mathrm{T}} \omega\right\}$, where $\mathrm{H}=\sum_{t=1}^{i} f(\mathbf{t}) \cdot f(\mathbf{t})^{\mathrm{T}}$ and $\Phi=-\sum_{t=1}^{i}[d(t) \cdot f(\mathbf{t})]$.

The optimal solution was obtained by solving the extremums of the Lagrange function, which was defined as

$$
L(\omega, \lambda)=\frac{1}{2} \boldsymbol{\omega}^{T} \mathrm{H} \boldsymbol{\omega}+\Phi^{T} \boldsymbol{\omega}-\lambda^{T}(A \omega-b),
$$


where $A$ and $b$ are linear constraints, $A$ is a matrix, $b$ is a column vector, and $\lambda$ is also a column vector. and the extremum condition was

$$
\left\{\begin{array}{l}
\frac{\partial L(\omega, \lambda)}{\partial \omega}=0 \\
\frac{\partial L(\omega, \lambda)}{\partial \lambda}=0
\end{array}\right.
$$

$\left[\begin{array}{cc}\mathrm{H}-\mathrm{A}^{\mathrm{T}} \\ -\mathrm{A} & 0\end{array}\right]\left[\begin{array}{l}\omega \\ \lambda\end{array}\right]=\left[\begin{array}{c}-\Phi \\ -\mathrm{b}\end{array}\right]$, where the matrix $\mathrm{A}$ was required to be invertible.

\section{Experiments and Discussion}

4.1. Displacement Tracking Performance. In the two PI models, the operator thresholds were divided into 16 groups from 0 to 150 at equal intervals. Based on the nonredundant principle, the repeated experiments were carried out to obtain the specific operator threshold. Four types of operators with thresholds of $0,10,30$, and 150 were used to implement the conventional PI model. The other four operators with thresholds of $0,20,40$ and 40 were used to implement the multislope PI model. The driving voltage was a triangular wave with $300 \mathrm{~s}$ period and amplitude $150 \mathrm{~V}$. Table 1 shows the identified weight parameters in the conventional PI model and the multislope PI method.

Figure 7 shows the experimentally measured hysteresis curve and the two fitted curves performed by two PI models. It was demonstrated that the improved PI model was more suitable for fitting the hysteresis curve. In particular, the conventional PI model cannot return to the origin point after eliminating the driving voltage.

The comparison of fitting errors between the two PI models is shown in Figure 8. For the conventional PI model, there were three regions with large fitting errors, namely, low-driving voltage stage, intermediate-driving voltage stage, and high-driving voltage stage. This phenomenon was consistent with Figures 5 and 6 . The extreme error in the low-driving voltage stage reached $-3.0900 \mu \mathrm{m},-2 \mu \mathrm{m}$, and $2.4 \mu \mathrm{m}$ in the abovementioned three regions, respectively. The maximum absolute error was $3.09 \mu \mathrm{m}$, and the mean absolute error was $0.9397 \mu \mathrm{m}$. While in the multislope PI model, the fitting error hovered between $-0.6 \mu \mathrm{m}$ and $0.6 \mu \mathrm{m}$, the maximum absolute error was $0.5850 \mu \mathrm{m}$, and the mean absolute error was $0.2013 \mu \mathrm{m}$, respectively. Compared with the mean absolute error, the multislope PI method was $78.6 \%$ less than the conventional PI model.

In order to better explain the improved multislope PI model, the mechanism for reducing the fitting error was discussed in the following. For the central symmetry of the PI operator, only the rising phase of driving voltage was analyzed.

As shown in equation (3), $y(\mathrm{t})$ was superposed by several operators. In the rising stage of the driving voltage, $r_{0}=0, y_{1}$, $y_{2}, y_{\mathrm{k}}$ can be expressed as

$$
\begin{aligned}
& y_{1}=\left(r_{1}-r_{0}\right) \tan \theta_{0}=r_{1} \tan \theta_{0}, \\
& y_{2}=r_{2} \tan \theta_{0}+\left(r_{2}-r_{1}\right) \tan \theta_{1},
\end{aligned}
$$

TABLE 1: Identified weight parameters in two models.

\begin{tabular}{ccccc}
\hline \multirow{k}{*}{$\begin{array}{c}\text { Conventional PI } \\
\text { Threshold }\end{array}$} & Weight & Threshold & Weight \\
\hline 0 & 0 & 0.008 & 0 & 0.196 \\
1 & 10 & 0.252 & 20 & 0.026 \\
2 & 30 & 0.019 & 30 & 0.046 \\
3 & 150 & 0.582 & 40 & 0.028 \\
\hline
\end{tabular}

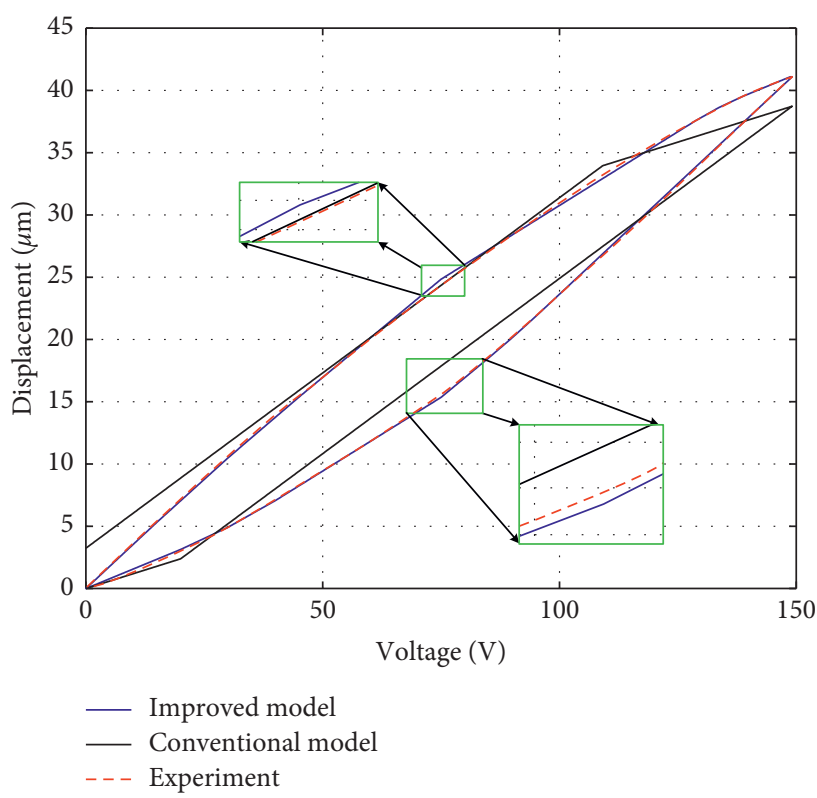

FIGURE 7: Comparisons of fitting curves with the experimental hysteresis curve.

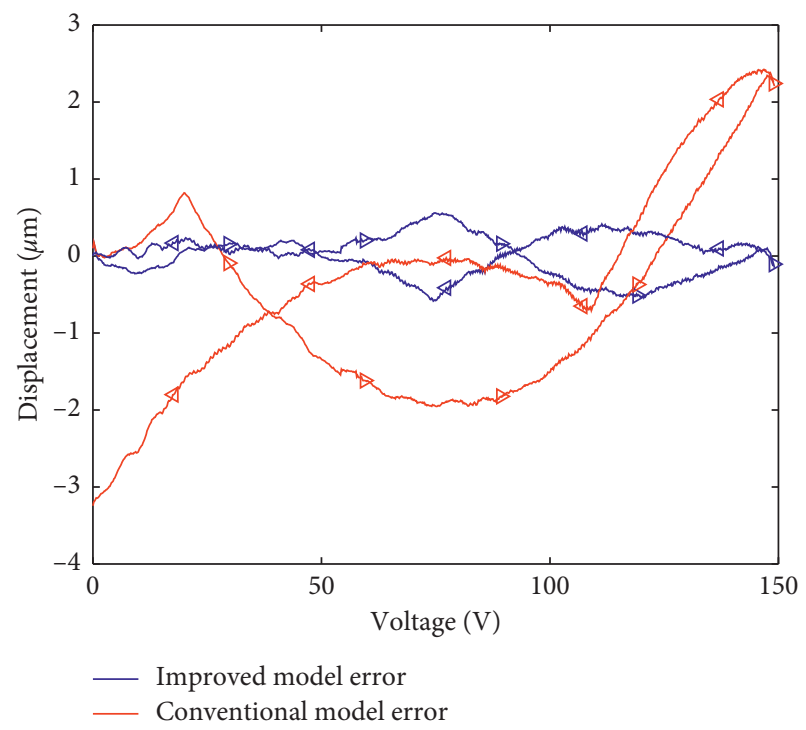

Figure 8: Comparison of fitting errors.

$$
\begin{aligned}
y_{k-1}= & r_{k-1} \tan \theta_{0}+\left(r_{k-1}-r_{1}\right) \tan \theta_{1}+\cdots \\
& +\left(r_{k-1}-r_{k-2}\right) \tan \theta_{k-2}
\end{aligned}
$$




$$
y_{k}=r_{k} \tan \theta_{0}+\left(r_{k}-r_{1}\right) \tan \theta_{1}+\cdots+\left(r_{k}-r_{k-1}\right) \tan \theta_{k-1},
$$

where $\theta_{k}=\arctan \omega_{k}$.

Equation (15) minus equation (14)

$$
y_{k}-y_{k-1}=\left(r_{k}-r_{k-1}\right)\left(\tan \theta_{0}+\tan \theta_{1}+\cdots+\tan \theta_{k-1}\right) \text {. }
$$

As shown in Figure 9, the following equations were easily deduced by geometric calculations:

$$
\begin{gathered}
y_{1}=\left(r_{1}-r_{0}\right) \tan \theta_{0}^{\prime}=r_{1} \tan \theta_{0}^{\prime}, \\
y_{2}=y_{1}+\left(r_{2}-r_{1}\right) \tan \theta_{1}^{\prime}, \\
y_{k}=y_{k-1}+\left(r_{k}-r_{k-1}\right) \tan \theta_{k-1}^{\prime} .
\end{gathered}
$$

According to equations (16) and (19), $\tan \theta_{k-1}^{\prime}=\tan \theta_{0}+\tan \theta_{1}+\cdots+\tan \theta_{k-1}$.

$$
\omega_{i}^{\prime}=\arctan \left(\tan \omega_{i}+\cdots+\tan \omega_{1}+\tan \omega_{0}\right) .
$$
(20):

The following discussion can be derived from equation

(1) As the threshold increases, the slope becomes larger and larger.

(2) The weight of the current operator was closely related to the previous weight coefficient. The larger the threshold number, the more weights were involved.

(3) The accuracy of fitting can be performed by increasing the number of operators, but correspondingly, the number of parameters also increased, making identification more difficult. In the conventional PI model or other improved PI models, the number of model operators is generally set to 4 .

Although the complexity of the multislope PI model is a little higher than that of the conventional PI model, the difference of computational cost between the multislope PI model and the conventional PI model is very small. This is because both the two PI models essentially consist of linear superposition mathematical operations, and the computational costs are all very small.

4.2. Feedforward Compensation Experiment. For verifying the positioning performance of the improved PI model, feedforward compensation was implemented, as shown in Figure 10. It was actually an open-loop system and its performance depended on the proposed PI method.

One of the reasons why the PI model was widely used was that its inverse model was easily available. The parameters of the PI model and its inverse model have the following relationships:

$$
r_{i}^{\prime}=\left.y(t)\right|_{t=r_{i}}=\sum_{j=1}^{i} \omega_{j}\left(r_{i}-r_{j}\right) \quad i=0,2, \cdots, n-1,
$$

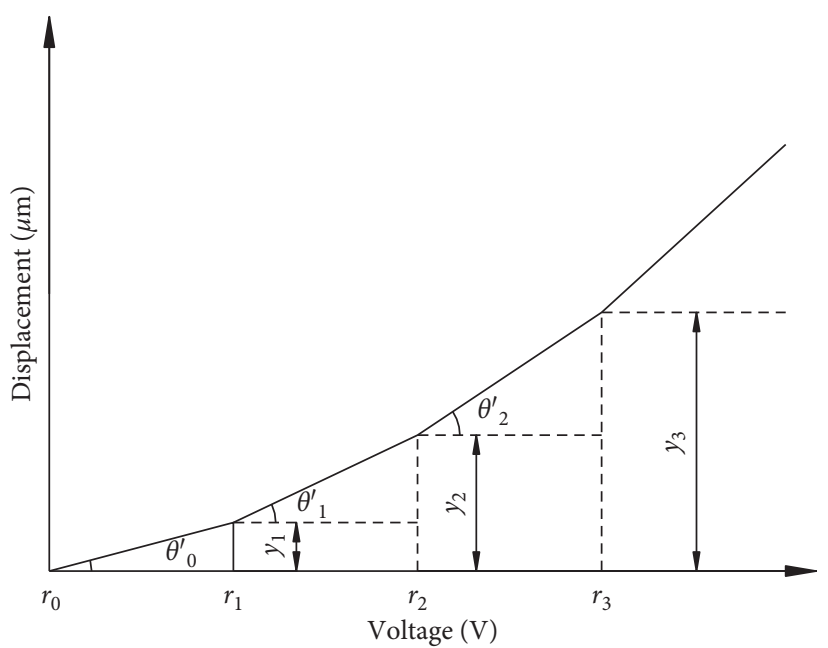

Figure 9: Mechanism of fitting error.

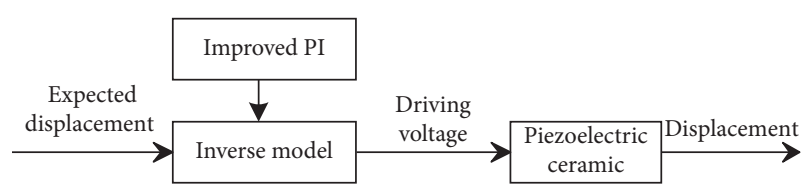

Figure 10: Control diagram of feedforward compensation.

where $r_{i}^{\prime}$ is the threshold of the PI inverse model.

For $\mathrm{dy}^{-1} / \mathrm{dr}_{i}^{\prime}=1 /\left(\mathrm{dy} / \mathrm{dr}_{i}\right)$, the relationship of slopes in the PI model and its inverse model

$$
\omega_{0}^{\prime}=\frac{1}{\omega_{0}} .
$$

It can be seen from equation (3) that dy $/ \mathrm{dr}_{i}=\sum_{j=0}^{i} \omega_{j}$. Similarly, $\mathrm{dy}^{-1} / \mathrm{dr}_{i}^{\prime}=\sum_{j=0}^{i} \omega_{j}^{\prime}$, and the slope can be written as

$$
\begin{aligned}
& \sum_{j=0}^{i} \omega_{j}^{\prime}=\frac{1}{\sum_{j=0}^{i} \omega_{j}}, \\
& \sum_{j=0}^{i-1} \omega_{j}^{\prime}=\frac{1}{\sum_{j=0}^{i-1} \omega_{j}} .
\end{aligned}
$$

By combining equations (23) and (24),

$$
\omega_{i}^{\prime}=-\frac{\omega_{i}}{\left[\left(\sum_{j=1}^{i} \omega_{j}\right)\left(\sum_{j=1}^{i-1} \omega_{j}\right)\right]} \quad i=1,3, \cdots, n-1 .
$$

The initial value of the operator in the PI inverse model was

$$
u_{i}(0)=\sum_{j=i}^{i-1} \omega_{j} f_{j}(0)+\sum_{j=1}^{n} \omega_{j} f_{j}(0) \quad i=2,3, \cdots, n .
$$

The expression of the multislope PI inverse model was 


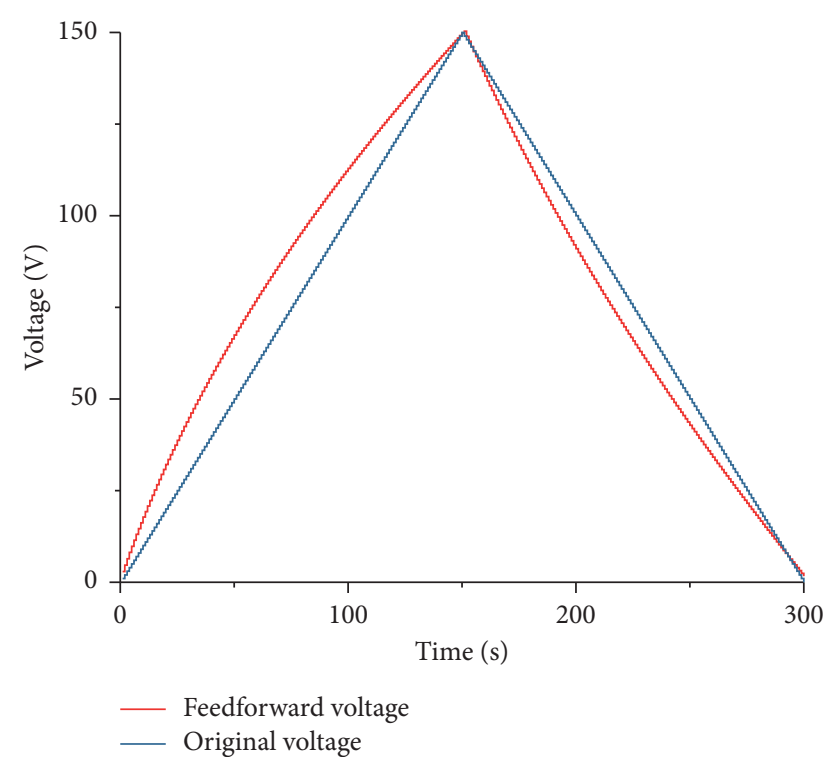

FIgURE 11: Comparisons of driving voltages.

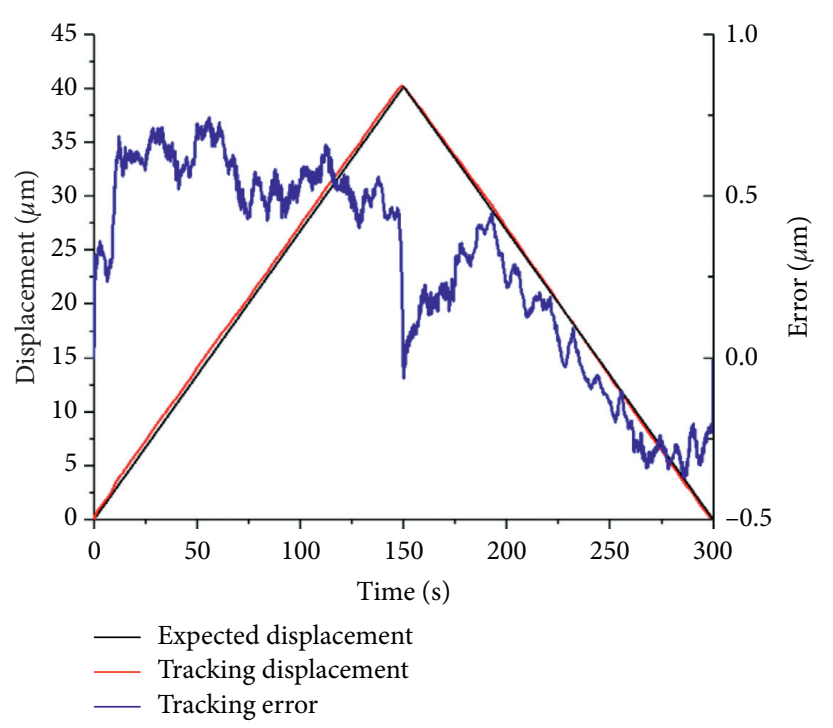

FIGURE 12: Feedforward compensation performance of the improved PI model.

$$
U(t)=\sum_{i=1}^{n} \omega_{i}^{\prime} u_{i}(t)=\sum_{i=1}^{n} \omega_{i}^{\prime} \cdot \max \left\{\begin{array}{c}
\max \left\{0.8\left[u(t)-r_{k}\right], 1.2 u(t)-r_{k}-30\right\}, \\
\min \left\{\min \left[1.2 u(t), 0.8 u(t)+30-0.6 r_{k}\right], y(t-T)\right\}
\end{array}\right\} .
$$

The driving voltage was a triangular wave with a $300 \mathrm{~s}$ period and amplitude $150 \mathrm{~V}$, as shown in Figure 11. During the increasing stage of driving voltage, the compensation effect gradually increased and then gradually decreased. This was mainly determined by the actual hysteresis curve of the piezoceramic beam. During the middle stage of the driving voltage, the hysteresis nonlinearity reached its maximum. The situation of the driving voltage decreasing stage was just opposite to the voltage increasing stage, and the analysis was similar to the aforementioned.

The feedforward compensation performance is shown in Figure 12. In the $0 \mathrm{~V}-150 \mathrm{~V}$ stage, the displacement error was basically around $0.5 \mu \mathrm{m}$. As the voltage increases, the displacement error firstly increased and then decreased. When the driving voltage reached $150 \mathrm{~V}$, the displacement error dramatically dropped to about $0 \mu \mathrm{m}$, indicating that the improved PI model fitted the endpoint of the hysteresis curve perfectly. During the $150 \mathrm{~V}-300 \mathrm{~V}$ stage, the displacement error was between $-0.3 \mu \mathrm{m}$ to $0.5 \mu \mathrm{m}$. As the voltage decreases, the error increased firstly and then decreased, which was consistent with the situation in the $0 \mathrm{~V}-150 \mathrm{~V}$ stage. The mean tracking error of the entire stage was $0.2828 \mu \mathrm{m}$, which was within $1 \%$ of the $45 \mu \mathrm{m}$ displacement range of the piezoceramic beam, and the average mean square error was $0.3100 \mu \mathrm{m}$, which demonstrated a good feedforward compensation control accuracy.

It can also be seen from Figure 12 that the compensation error would be relatively large in the middle part of the voltage rising and falling stages, which were consistent with Figure 8. By adding operators, the displacement error could be further reduced, but the task of parameter identification also increased dramatically.

\section{Conclusions}

(1) Based on the actual hysteresis curve of the piezoceramic beam, a multislope PI model was proposed, which essentially replaced the single-slope operators in the conventional PI method with multislope polyline operators. The theoretical analysis implied that the proposed multislope PI model could improve the fitting accuracy of the hysteresis curve compared with the conventional PI method.

(2) The feedforward compensation experiments indicated that the improved PI model has a mean tracking error of $0.2828 \mu \mathrm{m}$, within $1 \%$ of displacement full scale, and a mean square error of $0.3100 \mu \mathrm{m}$, which demonstrated a remarkable displacement tracking accuracy.

(3) By adding more operators, the control accuracy of feedforward compensation can be further improved, especially in the middle stages of the driving voltage, but the parameter identification would become more difficult.

\section{Data Availability}

The data used to support the findings of this study are available from the corresponding author upon request.

\section{Conflicts of Interest}

The authors declare that they have no conflicts of interest. 


\section{Acknowledgments}

This research was supported by the National Natural Science Foundation of China (Grant no. 51975171) and National key R\&D Program of China (Grant no. 2017YFB1301300).

\section{References}

[1] S. Zhang, F. Li, X. Jiang, J. Kim, J. Luo, and X. Geng, “Advantages and challenges of relaxor- $\mathrm{PbTiO} 3$ ferroelectric crystals for electroacoustic transducers-a review," Progress in Materials Science, vol. 68, pp. 1-66, 2015.

[2] G.-Y. Gu, L.-M. Zhu, C.-Y. Su, H. Ding, and S. Fatikow, "Modeling and control of piezo-actuated nanopositioning stages: a survey," IEEE Transactions on Automation Science and Engineering, vol. 13, no. 1, pp. 313-332, 2016.

[3] C. Yang, C. Li, and J. Zhao, "A nonlinear charge controller with tunable precision for highly linear operation of piezoelectric stack actuators," IEEE Transactions on Industrial Electronics, vol. 64, no. 11, pp. 8618-8625, 2017.

[4] M. Biggio, M. Butcher, A. Giustiniani, A. Masi, and M. Storace, "Memory characteristics of hysteresis and creep in multi-layer piezoelectric actuators: an experimental analysis," Physica B: Condensed Matter, vol. 435, no. 1, pp. 40-43, 2014.

[5] N. Jalili, Piezoelectric-Based Vibration Control: From Macro to Micro/Nano Scale Systems, Springer US, New York, NY, USA, 2010.

[6] I. D. Mayergoyz, "Dynamic Preisach models of hysteresis," IEEE Transactions on Magnetics, vol. 24, no. 6, pp. 2925-2927, 1988.

[7] D. Habineza, M. Rakotondrabe, and Y. Le Gorrec, "Bouc-wen modeling and feedforward control of multivariable hysteresis in piezoelectric systems: application to a 3-DoF piezotube scanner," IEEE Transactions on Control Systems Technology, vol. 23, no. 5, pp. 1797-1806, 2015.

[8] Y. Chen, Y. Chen, and C. Huang, "Nano-scale positioning design with piezoelectric materials," Micromachines, vol. 8, no. 12, p. 360, 2017.

[9] V. V. Aleshin and K. V. D. Abeele, "Preisach description for solids with frictional cracks," International Journal of NonLinear Mechanics, vol. 104, pp. 28-38, 2018.

[10] M. A. Janaideh and P. Krejčí, "Inverse rate-dependent Prandtl-ishlinskii model for feedforward compensation of hysteresis in a piezo micropositioning actuator," IEEE/ASME Transactions on Mechatronics, vol. 18, no. 5, pp. 1498-1507, 2013.

[11] T. Zhang, Q. Zhang, and Q. Wang, Model Detection for Functional Polynomial Regression, Elsevier Science Publishers B. V., Amsterdam, Netherlands, 2014.

[12] Y. Chen, J. Qiu, J. Palacios, and E. C. Smith, "Tracking control of piezoelectric stack actuator using modified Prandtl-Ishlinskii model," Journal of Intelligent Material Systems and Structures, vol. 24, no. 6, pp. 753-760, 2012.

[13] V. Hassani and T. Tjahjowidodo, "A hysteresis model for a stacked-type piezoelectric actuator," Mechanics of Advanced Materials and Structures, vol. 24, no. 1, pp. 73-87, 2017.

[14] G. Y. Gu, L. M. Zhu, and C. Y. Su, "Modeling and compensation of asymmetric hysteresis nonlinearity for piezoceramic actuators with a modified Prandtl-ishlinskii model," IEEE Transactions on Industrial Electronics, vol. 61, no. 3, pp. 1583-1595, 2014.

[15] T. A. Wei, P. K. Khosla, and C. N. Riviere, "Feedforward controller with inverse rate-dependent model for piezoelectric actuators in trajectory-tracking applications," IEEE/
ASME Transactions on Mechatronics, vol. 12, no. 2, pp. 134142, 2007.

[16] M.-J. Yang, C.-X. Li, G.-Y. Gu, and L.-M. Zhu, "Modeling and compensating the dynamic hysteresis of piezoelectric actuators via a modified rate-dependent Prandtl-Ishlinskii model," Smart Materials and Structures, vol. 24, no. 12, p. 125006, 2015.

[17] Y. R. Ko, Y. Hwang, M. Chae et al., "Direct identification of generalized Prandtl-Ishlinskii model inversion for asymmetric hysteresis compensation," Isa Transactions, vol. 70, 2017.

[18] L. Li, C.-X. Li, G. Gu, and L.-M. Zhu, "Positive acceleration, velocity and position feedback based damping control approach for piezo-actuated nanopositioning stages," Mechatronics, vol. 47, pp. 97-104, 2017.

[19] D. An, H. Li, Y. Xu, and L. Zhang, "Compensation of hysteresis on piezoelectric actuators based on tripartite PI model," Micromachines, vol. 9, no. 2, p. 44, 2018. 